\title{
Seasonal Betacoronavirus Antibodies' Expansion Post-BNT161b2 Vaccination Associates with Reduced SARS-CoV-2 VoC Neutralization
}

\author{
Stefania Dispinseri ${ }^{1}$. Ilaria Marzinotto ${ }^{2} \cdot$ Cristina Brigatti $^{2} \cdot$ Maria Franca Pirillo $^{3}$ - Monica Tolazzi ${ }^{1}$. \\ Elena Bazzigaluppi ${ }^{2} \cdot$ Andrea Canitano $^{3} \cdot$ Martina Borghi $^{4}$ - Alessandra Gallinaro ${ }^{3} \cdot$ Roberta Caccia $^{5}$. \\ Riccardo Vercesi ${ }^{5} \cdot$ Paul F. McKay $^{6} \cdot$ Fabio Ciceri $^{7,8} \cdot$ Lorenzo Piemonti $^{2,7}$. Donatella Negri ${ }^{4} \cdot$ Paola Cinque $^{5}$. \\ Andrea Cara $^{3} \cdot$ Gabriella Scarlatti $^{1} \cdot$ Vito Lampasona ${ }^{2} \mathbb{0}$
}

Received: 2 September 2021 / Accepted: 28 November 2021 / Published online: 9 January 2022

(c) The Author(s) 2021

\begin{abstract}
SARS-CoV-2 vaccination is known to induce antibodies that recognize also variants of concerns (VoCs) of the virus. However, epidemiological and laboratory evidences indicate that these antibodies have a reduced neutralization ability against VoCs. We studied binding and neutralizing antibodies against the Spike protein domains and subunits of the Wuhan-Hu-1 virus and its alpha, beta, delta VoCs and of seasonal betacoronaviruses (HKU1 and OC43) in a cohort of 31 health care workers prospectively followed post-vaccination with BNT162b2-Comirnaty. The study of sequential samples collected up to 64 days post-vaccination showed that serological assays measuring IgG against Wuhan-Hu-1 antigens were a poor proxy for VoC neutralization. In addition, in subjects who had asymptomatic or mild COVID-19 prior to vaccination, the loss of nAbs following disease could be rapid and accompanied by post-vaccination antibody levels similar to those of naïve vaccinees. Interestingly, in health care workers naïve for SARS-CoV-2 infection, vaccination induced a rapid and transient reactivation of pre-existing seasonal coronaviruses $\mathrm{IgG}$ responses that was associated with a subsequent reduced ability to neutralize alpha and beta VoCs.
\end{abstract}

Keywords COVID-19 $\cdot$ Vaccine $\cdot$ Antibodies $\cdot$ Neutralizing antibodies

\section{Introduction}

Large-scale SARS-CoV-2 vaccination campaigns are ongoing although with large disparities between countries [1]. While antibodies to SARS-CoV-2 are not the only determinant of immunity, they are important predictors of

Stefania Dispinseri and Ilaria Marzinotto share the first authorship in this paper.

Gabriella Scarlatti

scarlatti.gabriella@hsr.it

Vito Lampasona

Lampasona.vito@hsr.it

1 Viral Evolution and Transmission Unit, IRCCS Ospedale San Raffaele, 20132 Milan, Italy

2 Diabetes Research Institute, IRCCS Ospedale San Raffaele, 20132 Milan, Italy

3 National Center for Global Health, Istituto Superiore Di Sanità, 00161 Rome, Italy immunological protection from SARS-CoV-2 infection or disease [2]. However, the continuous worldwide emergence of SARS-CoV-2 variants of concern ( $\mathrm{VoC})$ is challenging the effectiveness of licensed vaccines with several open questions remaining about the effectiveness and persistence of vaccine-induced antibodies [3, 4]. Public health policies need to adapt on a rolling basis and several governments are already introducing additional rounds of vaccination in subjects at risk of severe disease [5]. Moreover, the contribution

4 Department of Infectious Diseases, Istituto Superiore Di Sanità, 00161 Rome, Italy

5 Unit of Infectious Diseases, IRCCS Ospedale San Raffaele, 20132 Milan, Italy

6 Department of Infectious Disease, Imperial College, London, UK

7 School of Medicine and Surgery, Università Vita-Salute San Raffaele, 20132 Milan, Italy

8 Hematology and Bone Marrow Transplantation Unit, IRCCS Ospedale San Raffaele, 20132 Milan, Italy 
to community immunity of subjects who experienced an asymptomatic or mild COVID-19 is unclear.

To address some of these issues, in this study, we analyzed prospectively the antibody response to SARS-CoV-2 and seasonal betacoronaviruses induced by BNT162b2Comirnaty vaccination in a cohort of health care workers (HCW) with or without a prior COVID-19. Overall, our results suggest that (1) vaccine-induced Wuhan-Hu-1 RBD IgGs should be used with caution as proxy for VoC neutralization; (2) in subjects who had asymptomatic or mild COVID-19, the loss of neutralizing antibodies (nAbs) following disease can be rapid and accompanied by antibody levels post-vaccination no greater than in naïve vaccinees, suggesting that protection from re-infection mediated by antibodies would be no better than in naïve subjects; and (3) we observed the reactivation of pre-existing seasonal coronaviruses' antibody responses following BNT162b2Comirnaty vaccination.

\section{Methods}

\section{Study Approval}

All procedures involving human participants were in accordance with the ethical standards of the institutional and/or national research committee and with the $1964 \mathrm{Hel}-$ sinki declaration and its later amendments or comparable ethical standards. Written informed consent was obtained from all individual participants included in the study.

The study subjects belonged to the IRCCS Ospedale San Raffaele COVID-19 cohort study COVID-BioB (registered as ClinicalTrialsgov-NCT04318366) approved by the IRCCS Ospedale San Raffaele Ethics Review Board (protocol 68/INT/2020) and to the COVID-BioB related immunological sub-study "Role of the immune response in the infection with SARS-CoV-2 and in the pathogenesis of COVID-19" (protocol number 34/INT/2020).

\section{Study Population}

The study subjects included 31 hospital health care workers (HCW) of the IRCCS Ospedale San Raffaele, who received their first dose of the Pfizer-BioNtech mRNA vaccine BNT162b2-Comirnaty between January 7th and March 5th, 2021. All but two subjects received their second vaccination jab after 21 days according to Italian national guidelines (https://www.gazzettaufficiale.it/atto/serie_generale/ caricaDettaglioAtto/originario?atto.dataPubblicazioneGaz zetta $=2021-03-24 \&$ atto.codiceRedazionale $=21 \mathrm{~A} 01802 \&$ elenco30giorni=false) (Supplemental Table S1). Thirteen were naïve to COVID-19 at vaccination, while 18 had a previous SARS-CoV-2 infection either during the first pandemic wave, between February and March 2020 ( $n=13$, median interval from symptoms onset to first jab 302.5 days), or during the second wave, between October and November $2020(n=5$, median interval from symptoms onset to first jab 76 days). Four individuals with COVID19 with a confirmed negative nasopharyngeal swab postinfection tested positive at a routine in-hospital screen 1.5 to 8 months later likely due to asymptomatic re-infection events.

A confirmed infection case was defined as a SARSCoV-2-positive real-time reverse-transcriptase polymerase chain reaction (RT-PCR) from a nasopharyngeal swab and/or COVID-19 symptoms and/or a serologic evidence of antibody to SARS-CoV-2. The WHO classification was used to define disease severity (https:// www.who.int/publications-detail-redirect/WHO-2019nCoV-clinical-2021-1). Asymptomatic infections were captured thanks to the hospital SARS-CoV-2 testing policy, which introduced in May 2020 a serologic antibody test (LIAISON® SARS-CoV-2 S1/S2 IgG serological test, DiaSorin S.p.A., Vercelli, Italy) and in August 2020 a regular monthly or bimonthly SARSCoV-2 nasal-pharyngeal swab test. The clinical and laboratory data were collected from medical chart review or directly by interview, crosschecked for accuracy by data managers and clinicians, and entered in a dedicated electronic case record form (eCRF) developed on site for the COVID-BioB study in MySQL (V5.7.14) on Apache Tomcat (V2.4.23) platform running on windows sever 2012 R2.

Biological material of the vaccinees included a dedicated serum sample collected at first vaccination and thereafter at 10,21,31, 42, and 64 days (with an approximation of \pm 5 days). All serum samples were coded and anonymously processed for the immunological studies.

\section{Construction of Plasmids for Lentiviral Pseudotypes' Expression of Variant Spikes}

A schematic representation of recombinant antigens used in this study is shown in Supplemental Figure S1. Briefly, the plasmid pSpike-C3 encodes a codon optimized SARSCoV-2 Spike protein open reading frame (ORF) (GenBank: NC_045512.2) containing a 21 amino acid deletion at the cytoplasmic tail (delta21) of Spike protein as previously described [6]. For construction of pSpike-UKC3 and pSpike-SAC3 plasmids, expressing the variant Spike ORFs with a 21 amino acid deletion at the cytoplasmic tail, a NheI/AvaI fragment of DNA was removed from Alpha (B.1.1.7 pSpike-UK) and Beta (B.1.351 pSpike-SA) plasmids and inserted into the corresponding restriction sites of pSpike-C3 plasmid, to obtain pSpike-UKC3 and 
pSpike-SAC3 plasmids. Plasmid pSpike-INd19 was constructed by inserting a codon optimized B.1.617.2 Spike (Delta) sequence with a 19 amino acid deletion at the cytoplasmic tail into HindII/NotI restriction sites of pcDNA3.1 expression plasmid. The B.1.1.7 Alpha pSpike-UKC3 used in these studies contained the following mutations: del6970HV, del145Y, N501Y, A570D, D614G, P681H, T716I, S982A, D1118H. The B.1.351 Beta pSpike-SAC3 used in these studies contained the following mutations: L18F, D80A, D215G, del242-244LAL, R246I, K417N, E484K, N501Y, D614G, A701V. The B.1.617.2 Delta pSpikeINd19 used in these studies contained the following mutations: T19R, del157-158, L452R, T478K, D614G, P681R, D950N (Supplemental Fig. S1).

\section{Production of a Simian Lentiviral Vector Expressing Luciferase (LV-Luc) and Pseudotyped with Spike Variants}

293 T Lenti-X human embryonic kidney cell line (Clontech, Mountain View, CA, USA) was used for production of LV-Luc pseudotyped with Spike variants by transient transfection as previously described [6]. Briefly, $293 \mathrm{~T}$ Lenti-X cells $(3.5 \times 106$ cells $)$ were seeded on $10-\mathrm{cm}$ Petri dishes (Corning Incorporated-Life Sciences, Oneonta, NY, USA) and transiently transfected with plasmid pGAE-LucW, pADSIV3 + and pseudotyping plasmid (pSpike-C3, pSpike-UKC3, pSpike-SAC3, pSpkeINd19) using the JetPrime transfection kit (Polyplus Transfection Illkirch, France) following the manufacturer's recommendations using a 1:2:1 ratio (transfer vector:packaging plasmid:Spike plasmid). At $48 \mathrm{~h}$ post-transfections, culture supernatants containing the LV-Luc pseudotypes (LV-Luc/ Spike-C3, LV-Luc/Spike-UKC3, LV-Luc/Spike-SAC3, and LV-Luc/Spike-INd19) were collected and stored in 1-mL aliquots at $-80^{\circ} \mathrm{C}$ until use.

\section{Lentiviral Vector-Based SARS-CoV-2 Neutralization Assay}

LV-Luc preparations were titered on VeroE6 cells (African green monkeys, epithelial kidney). Dilutions providing 150,000-200,000 relative luciferase units (RLU) were used in the neutralization assay, as previously described [6]. Briefly, heat-inactivated serum serial threefold dilutions starting from the 1/40 dilution were incubated in duplicate with the LV-Luc for $30 \mathrm{~min}$ at $37{ }^{\circ} \mathrm{C}$ in 96 -well plates, and thereafter added to VeroE6 cells at a density of 20,000 cells/ well. After $48 \mathrm{~h}$, luciferase expression was determined with a luciferase assay system (Bright-Glo, Promega) and measured in a Mitras luminometer (Berthold, Germany). The 50\% inhibitory serum dilution (ID50) was calculated with a linear interpolation method using the mean of the duplicates [7]. Neutralization was expressed as the reciprocal of the serum dilution giving $50 \%$ inhibition of RLU compared to the mean of the virus control wells. An ID50 below 1/40 serum dilution was considered negative and a value of 10 ascribed for statistical analysis.

\section{IgG Binding Antibody Luciferase Immunoprecipitation System (LIPS) Assay}

Using LIPS [8], we measured IgG binding to recombinant nanoluciferase tagged antigens corresponding to the following: SARS-CoV-2 Wuhan-Hu-1, alpha, beta, and delta spike RBD domains; Wuhan-Hu-1 spike S2 subunit; HKU1 and OC43 seasonal betacoronaviruses' spike S1 B domain and spike S2 subunits; pandemic Ca2009 H1N1 influenza virus hemagglutinin (HA) antigen, as previously described $[9,10]$ (Supplemental Figure S1). Viral sequences used in this study correspond to the following deposited sequences: GenBank NC_045512.2 for SARS-CoV-2 Wuhan, GenBank AGW27863.1 for HKU1 [11], MZ450972.1 for OC43. HKU1 and OC43 viral sequences correspond to isolates reported multiple times in the USA, China, and Japan over the last decade. Briefly, we cloned recombinant nanoluciferase-tagged monomeric or multimeric antigens and expressed them by transient transfection into Expi293F cells (Expi293 ${ }^{\mathrm{TM}}$ Expression System, Thermo Fisher Scientific Life Technologies, Carlsbad, CA, USA). For LIPS, we incubated in liquid phase each antigen with test serum (1ul) for $2 \mathrm{~h}$ and then captured immune-complexes with rProtein A-sepharose. After washing (5 times) the sepharose pellets, we quantified bound $\mathrm{IgG}$ by measuring the recovered luciferase activity in a Berthold Centro XS3 luminometer (Berthold Technologies GmbH \& Co. KG, Bad Wildbad, Germany) using the MikroWin version 5.22 software. We then converted raw data into arbitrary units (AU); for SARSCOV-2-specific antibodies, we used a local positive index serum that exhibited closely similar binding to each of the VoCs, and for HKU1- and OC43-specific antibodies, we used two local index sera with strong antibody binding to the respective betacoronavirus antigen.

\section{Statistics}

We performed statistical analyses using the $\mathrm{R}$ software version 3.4.1 [12]. We reported the median with either $95 \% \mathrm{CI}$, range, or interquartile range (IQR) for continuous variables and frequency or percent $\%$ frequency for categorical variables. We used repeated measures ANOVA to compare continuous variables over time after log transformation. Two-tailed $p$ values are reported, with $p$ value $<0.05$ indicating statistical significance. 


\section{Results}

We used validated lentiviral vector-based SARS-CoV-2 neutralization and LIPS assays [6,9] to profile the antibody response to spike antigens of SARS-CoV-2 (Wuhan-Hu-1, alpha, beta, and delta VoCs) and of seasonal betacoronaviruses (HKU1 and OC43) post-BNT162b2-Comirnaty vaccination (Supplemental Figure S1). We tested sera from 31 vaccinated HCW of the IRCCS Ospedale San Raffaele, Milan, Italy, with $(n=18)$ or without $(n=13)$ prior confirmed COVID-19 (Supplemental Table S1). All but two subjects received two vaccine doses 21 days apart, between January 7th and March 5th, 2021. We collected serum samples prospectively at first vaccination (baseline), and thereafter at 10, 21, 31, 42, and 64 days.

\section{Binding and Neutralizing Antibodies Against Wuhan-Hu-1 Antigens in BNT162b2 Vaccinees Stratified by SARS-CoV-2 Antibody Status at Baseline}

At baseline before vaccination, SARS-CoV-2 Wuhan-Hu-1 nAbs and IgGs binding to spike RBD were absent in 6 out of $18 \mathrm{HCW}$ with a prior history of COVID-19 and in all those without. All vaccinees responded to BNT162b2-Comirnaty immunization by producing antibodies able to both neutralize SARS-CoV-2 Wuhan-Hu-1 and bind its RBD and S2 domains (Supplemental Figure S2, A-C). Antibody binding to the Wuhan-Hu-1 spike RBD and S2 domains and neutralization titer increased above assay thresholds starting from day 10 post-vaccination. We observed a peak of antibody
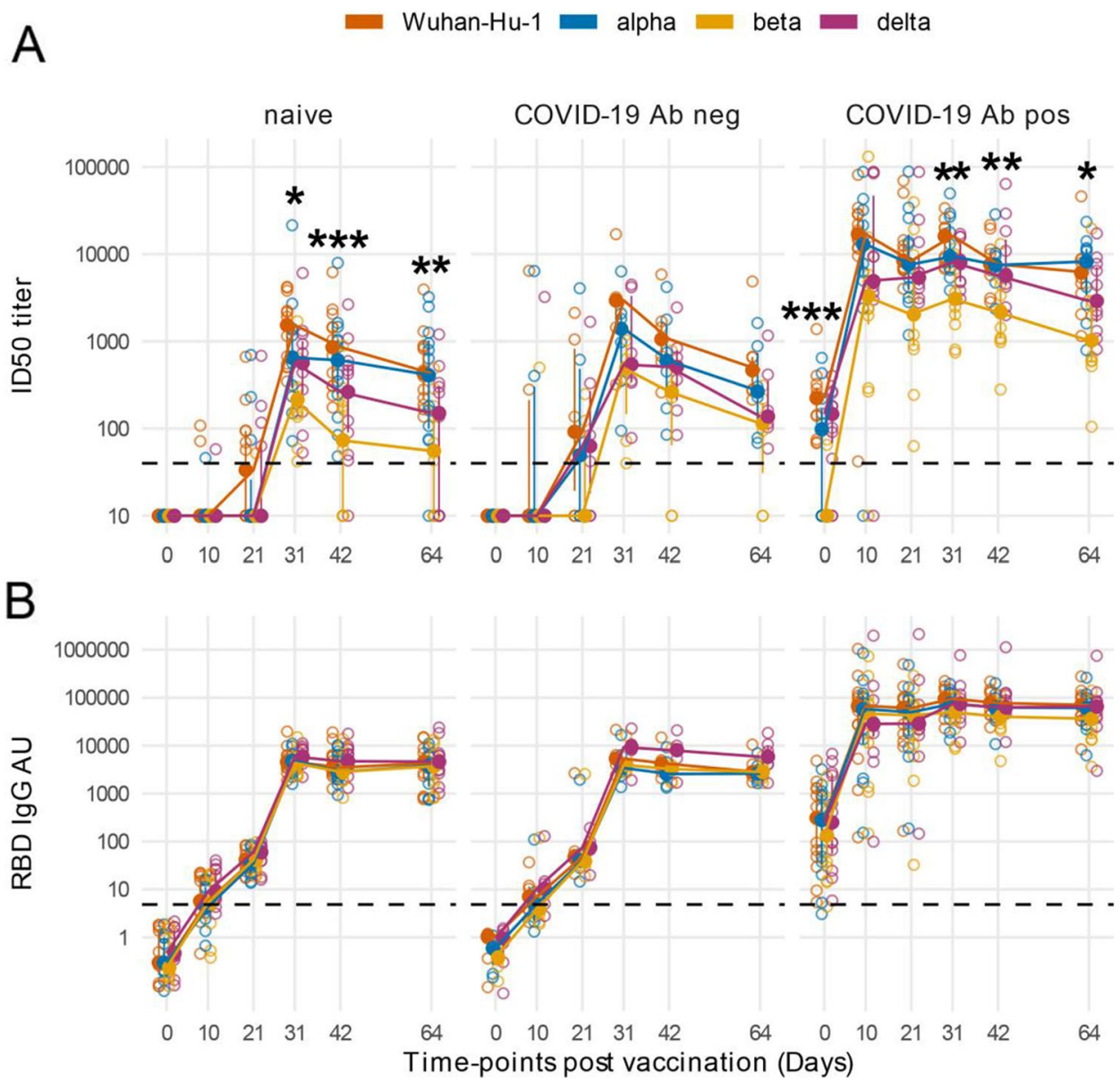

Fig. 1 Antibody responses to Spike antigens of SARS-CoV-2 variants post-BNT162b2 vaccination show significant differences in nAbs titer but not in IgG binding to the RBD of VoCs. Line plots of ID50 titer (A) and RBD IgG arbitrary units (AU) (B) against SARS-CoV-2 Wuhan-Hu-1 and VoCs in sequential samples after vaccination. Vaccinees are presented in left to right panels stratified as follows: (left panel) subjects naïve for SARS-CoV-2 infection $(n=13)$, (middle panel) with prior confirmed COVID-19 presenting at vaccination either without Wuhan-Hu-1 nAbs and RBD IgGs $(n=6)$, and (right panel) with prior COVID-19 and SARS-CoV-2 antibodies at baseline $(n=12)$. Filled circles and bars represent the median \pm inter quartile range at each time-point, and empty circles show each individual measurement. The horizontal dashed lines stand for the threshold for positivity. The asterisks indicate statistically significant differences in mean titers of nAbs or RBD IgGs levels across VoCs at the corresponding time-points (two-way repeated measures ANOVA, $* p$ adjusted $<0.05, * * p$ adjusted $<0.01, * * * p$ adjusted $<0.001$ ) 
responses already at day 10 in HCW with prior COVID-19 and detectable SARS-CoV-2 antibodies at baseline. In HCW naïve for COVID-19 or without SARS-CoV-2 antibodies at baseline, the antibody response peaked instead at day 31 , i.e., 10 days after the booster jab. Peak values were higher in $\mathrm{HCW}$ who presented with SARS-CoV-2 antibodies at baseline for both nAbs (ID50 geometric mean titer to Wuhan$\mathrm{Hu}-1$ at day 31 : in baseline antibody positive $\mathrm{HCW}=13,613$; in baseline antibody negative $\mathrm{HCW}=1607$ ) and $\mathrm{IgG}$ to RBD and S2 spike domains (Supplemental Table S2).

\section{Post-vaccination, the Antibody Response Shows Significant Differences in nAb Titer but Not in IgG Binding to SARS-CoV-2 VoCs}

NAbs and RBD IgGs against SARS-CoV-2 alpha, beta, and delta VoCs had post-vaccination kinetics similar to that against Wuhan-Hu-1, with delayed and lower peak titers in COVID-19 vaccinees without detectable antibodies at baseline (Fig. 1A, and B). NAb peak titers against VoCs were reduced compared with nAbs to Wuhan-Hu-1. The reduction of peak $\mathrm{VoC}$ beta nAb titer was statistically significant both in HCW naïve for COVID-19 or who presented with SARS-CoV-2 antibodies at baseline (Fig. 1A and Supplemental Table S2). cination, antibody levels to and its variants are correlated to previous disease severity. The line plots show the moving average of antibody levels against the indicated SARSCoV-2 variants after fitting of a LOESS polynomial regression. Subjects are stratified according to symptoms after SARS-CoV-2 infection. Antibody responses to VoCs post-BNT162b2 vaccination show differences in nAbs titer (A) but not in IgG binding to the RBD $(\mathbf{B})$
Fig. 2 Post-BNT162b2 vacspike antigens of SARS-CoV-2 Spike antigens of SARS-CoV-2

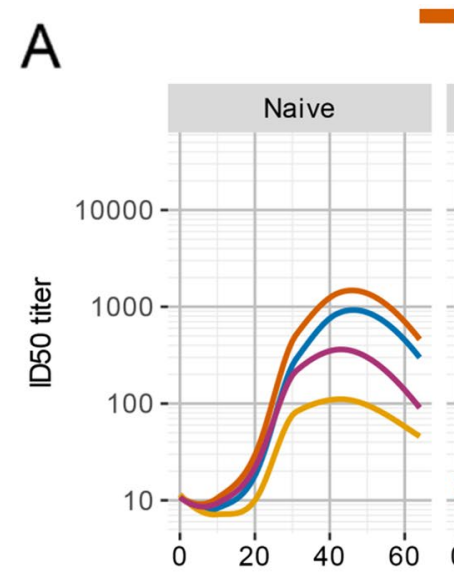

NAbs declined already from day 42 after the vaccination in HCW without nAbs at baseline (day 64/day $31 \mathrm{nAb}$ geometric mean titer ratio in $\mathrm{HCW}$ naïve: $0.37,0.43,0.23$, and 0.19 ; in HCW nAb negative: $0.23,0.30,0.22$, and 0.27 , for Wuhan-Hu-1, alpha, beta, and delta respectively) (Supplemental Table S2). At the end of follow-up, nAbs dropped below the threshold for positivity in $7 \mathrm{HCW}$ against the VoC beta (all of whom were antibody negative at baseline: 5 naïve for SARS-CoV-2 infection and 2 with prior COVID19) and in 4 against the VoC delta (all naïve for SARSCoV-2 infection). Conversely, IgG binding to Wuhan-Hu-1 and VoC RBD domains was comparable and showed only a modest drop by day 64 (Fig. 1B).

\section{Post-vaccination, Antibody Levels to Spike Antigens of SARS-CoV-2 and Its Variants Are Correlated to Previous Disease Severity}

HCW with prior COVID-19 were stratified according to disease severity: disease was asymptomatic in 5, mild in 11, and moderate in 2 (Supplemental Table S1). After BNT162b2 vaccination, we observed that $\mathrm{nAb}$ peak values increased according to disease severity in $\mathrm{HCW}$ (Fig. 2A). The reduction of $\mathrm{nAb}$ titer against $\mathrm{VoCs}$ was particularly evident in naïve subjects or who previously had asymptomatic or mild
Wuhan-Hu-1 alpha beta delta
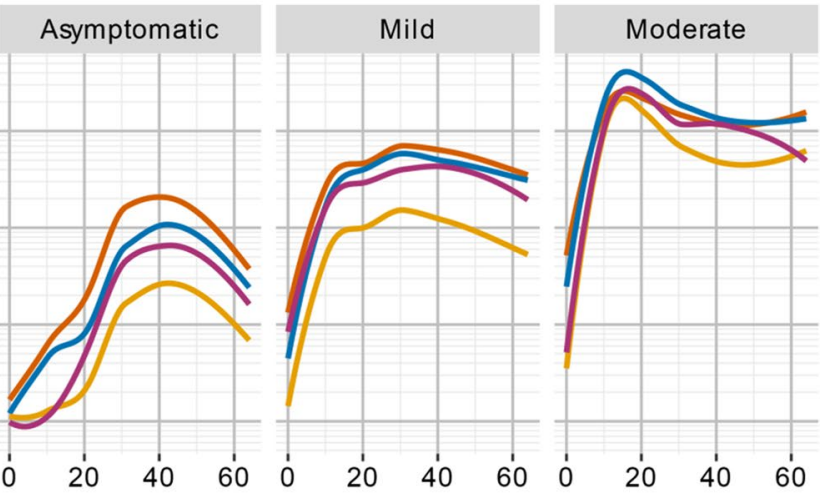
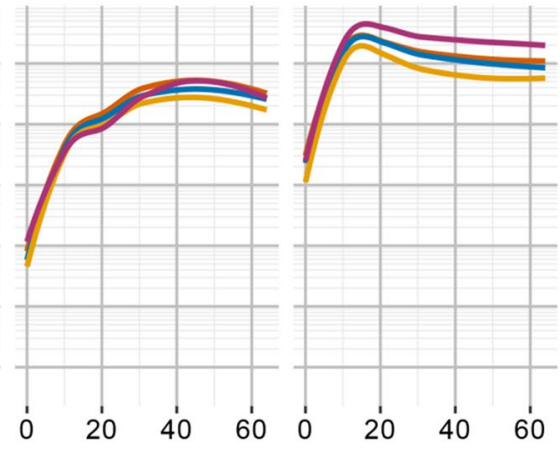

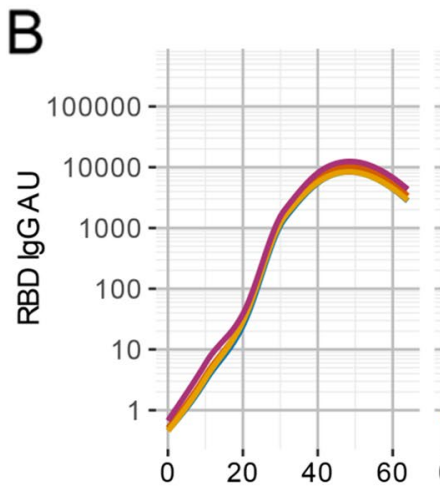

Days post vaccination 


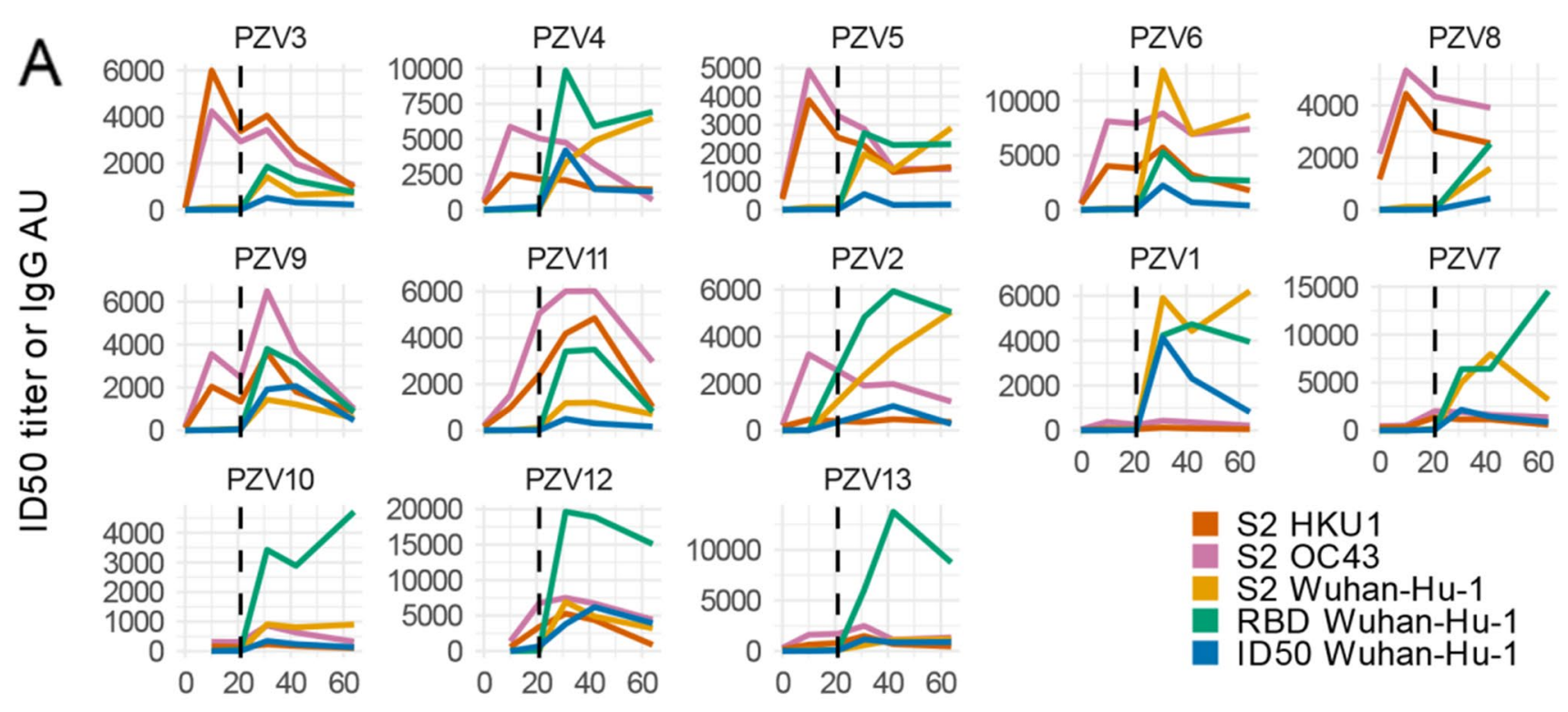

B
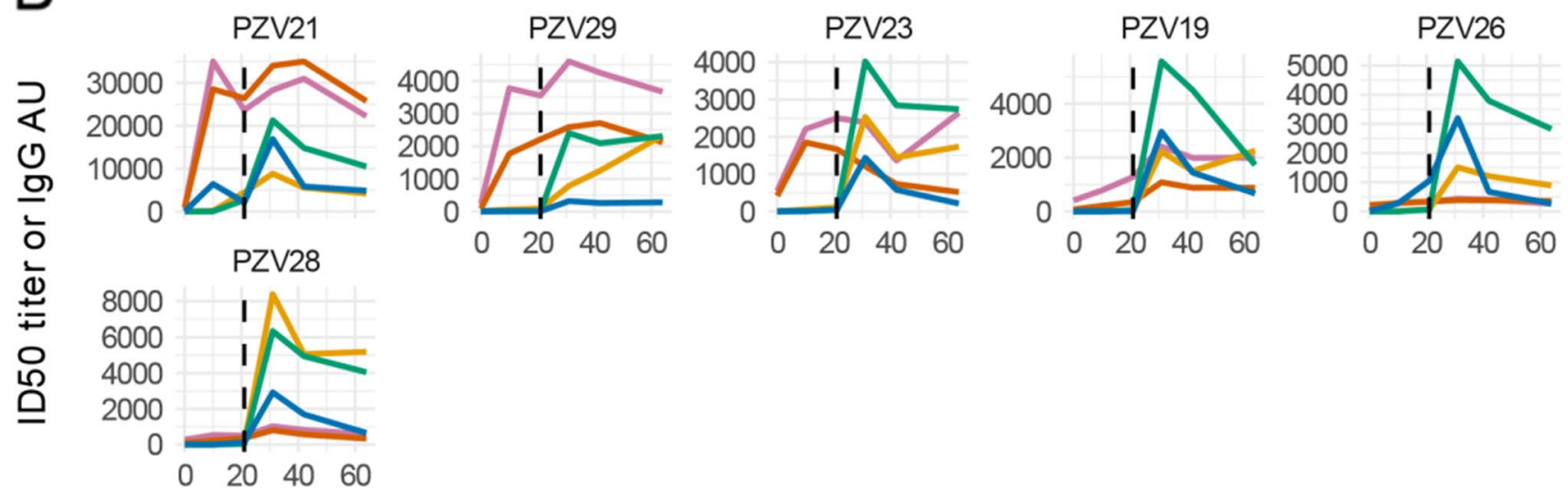

C
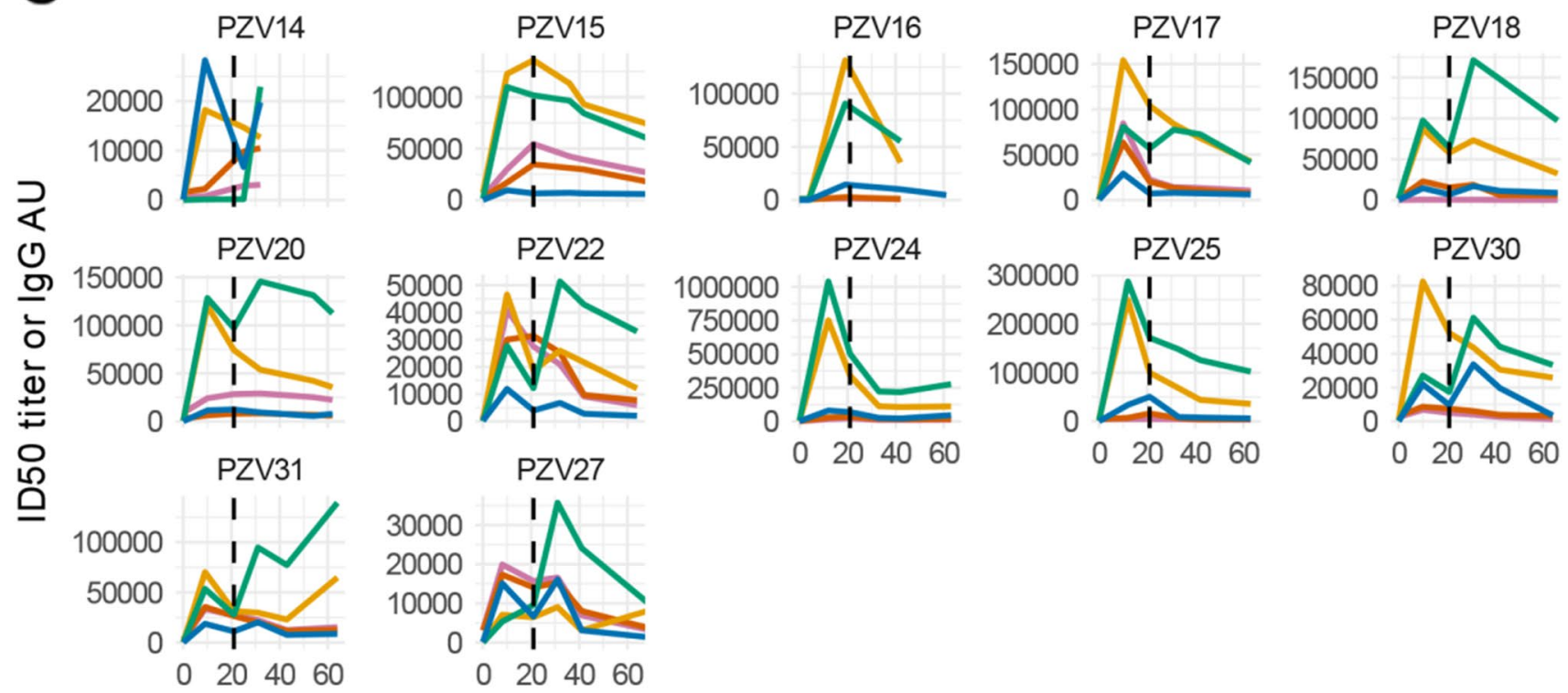

Days post vaccination 
4Fig. 3 BNT162b2-Comirnaty vaccination can induce a large, early boost of antibodies against seasonal betacoronaviruses in COVID19-naïve subjects. Line plots of ID50 nAbs and RBD IgG arbitrary units (AU) to SARS-CoV-2 Wuhan-Hu-1 and S2 spike proteins of SARS-CoV-2, OC43, and HKU1 betacoronaviruses at sequential time-points after vaccination. Vaccinees are stratified as follows: (A) subjects naïve for SARS-CoV-2 infection $(n=13)$, (B) subjects with prior confirmed COVID-19 presenting at vaccination either without Wuhan-Hu-1 nAbs and RBD IgGs $(n=6)$, and (C) with prior COVID-19 and SARS-CoV-2 antibodies at baseline $(n=12)$. The vertical dashed line indicates the 2 nd vaccine jab

symptoms. RBD IgG arbitrary units also showed a correlation with previous disease severity but the difference in peak IgG binding between Wuhan-Hu-1 spike and VoCs antigens within each $\mathrm{HCW}$ category was not significant (Fig. 2B).

\section{An Early Boost of HKU1 IgGs Post-vaccination Is Associated with a Rapidly Decreasing Ab Response Against SARS-CoV-2 Antigens in COVID-19-Naïve Subjects}

Vaccination induced a large, early boost of IgG against the extracellular domain of the OC43 and HKU1 seasonal betacoronaviruses' Spike S2 subunits in a fraction of HCW. The rise of seasonal coronaviruses Spike S2 antibodies occurred already at day 10 after the first vaccination in 7 out of 13 naïve HCW and 3 of $6 \mathrm{HCW}$ with prior COVID-19 (all SARS-CoV-2 Abs negative at baseline) (Fig. 3A, and B). In all $\mathrm{HCW}$ who presented with SARS-CoV-2 Abs at baseline, the $\mathrm{Ab}$ response against Wuhan-Hu-1 spike antigens at day 10 invariably exceeded that against seasonal betacoronaviruses S2 (Fig. 3C). The expanded IgG response to HKU1 and OC43 S2 antigens appeared to be minimally if at all cross-reactive with SARS-CoV-2 S2. No increase in IgG binding was instead observed against the Spike S1 B domain region of both seasonal betacoronaviruses that corresponds to the RBD on SARS-CoV-2 (Supplementary Figures S3-S4). This was consistent with the greater aminoacid sequence homology between seasonal beta coronaviruses and SARS-CoV-2 Spike S2 extracellular domains compared to the Spike S1 B and RBD domains (Supplementary Figures S5-S6).

To exclude a generalized impact of vaccination on preexisting antibody responses, we measured IgG binding to the HA antigen of the 2009 pandemic H1N1 flu virus in COVID-19-naïve HCW. HA antibodies showed modest fluctuations during follow-up, which were not synchronous with those against betacoronaviruses antigens (Supplemental Figure S7).

In SARS-COV-2-naïve HCW, HKU1 S2 IgGs above the median levels at day 10 post-vaccination were associated with a lower peak and a faster decline during followup of SARS-CoV-2 nAbs against Wuhan-Hu-1, alpha, and beta variants (two-way repeated measures ANOVA $p$ adjusted $<0.015$ ) (Fig. 4A). A similar trend was observed also for nAbs against the delta variant but did not reach statistical significance. At day 64 post-vaccination, in the 7 naïve subjects with an early boost of HKU1 antibodies, nAbs dropped below detection limit in 4 against the beta $\mathrm{VoC}$ and in 3 against the delta $\mathrm{VoC}$, compared to 1 of 6 of those without the early boost of seasonal betacoronaviruses antibodies. A similar but statistically not significant trend was present also in HCW with prior COVID-19 who presented at vaccination without SARS-CoV-2 antibodies (Supplemental Figure S8A). No differences in nAbs against the variants were present in HCW who at baseline had detectable SARSCoV-2 antibodies (Supplemental Figure S9A).

Similarly, always in HCW COVID-19 naïve with an early boost of HKU1 antibodies, we observed during follow-up a modest but significant peak reduction and decline of IgG against the SARS-CoV-2 RBD that reached statistical significance for Wuhan-Hu-1, alpha, and beta variants (two-way repeated measures ANOVA $p$ adjusted $<0.05$ ) (Fig. 4B). Stratification according to HKU1 IgG boost of HCW with prior COVID-19 was not associated with significant differences in SARS-CoV-2 RBD IgGs during follow-up (Supplementary Figures S8B-S9B).

\section{Discussion}

The results of our study indicate that vaccinees naïve for COVID-19 frequently show a rapid drop below detection limit of antibodies that cross-neutralize VoCs in vitro. Interestingly, a similar profile of post-vaccination antibodies was seen in some vaccinees who recovered from prior asymptomatic or mild COVID-19 and were Wuhan-Hu-1 nAbs negative at the time of vaccination. This was at variance with the outcome in vaccinees with prior COVID-19 with detectable Wuhan-Hu-1 nAbs at baseline, who had a neutralizing response an order of magnitude greater and sustained throughout follow-up. While the results of our in vitro test do not entirely recapitulate the ability of the vaccinees' immune system to stave off SARS-CoV-2 infection, this rapid decrease of antibodies able to neutralize VoCs is consistent both with the recently reported drop of vaccine effectiveness after the delta variant began to widely circulate and increased viral load in vaccinated individuals infected by VoCs [13].

Moreover, our study shows that the $\mathrm{nAb}$ response kinetics is not closely mirrored by that of IgG binding to the RBD. IgG binding to the Wuhan-Hu-1 and VoC RBDs was similar and showed only a modest drop by the end of the followup. This observation agrees with the recent evidence that many RBD IgGs in vaccinees are non-neutralizing and that several nAbs target the spike protein NTD rather than the 

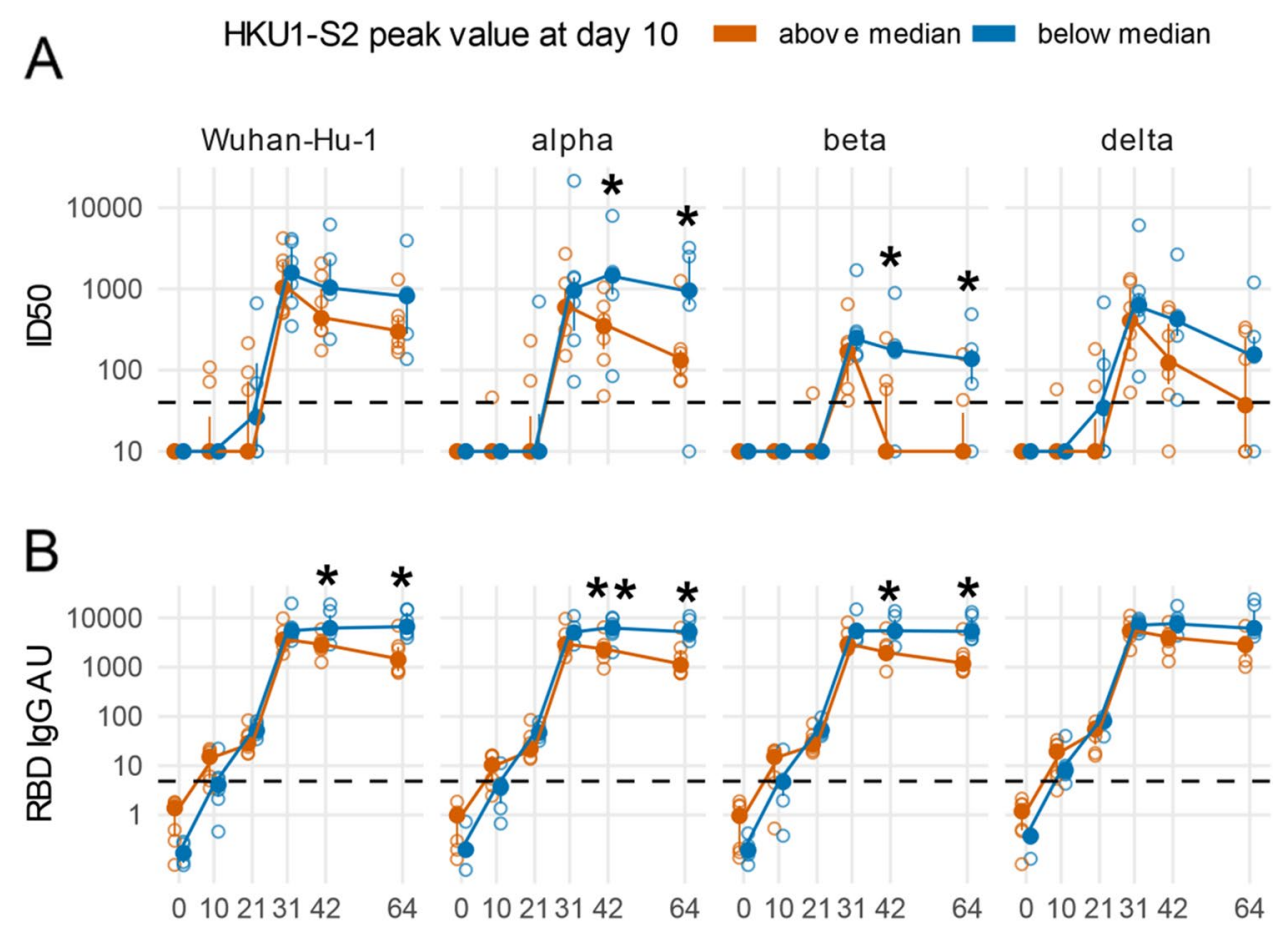

Time-points post vaccination (days)

Fig. 4 An early boost of HKU1 IgGs post-vaccination is associated with a rapidly decreasing $\mathrm{Ab}$ response against SARS-CoV-2 in COVID-19-naïve subjects. Line plots of Wuhan-Hu-1 and indicated VoCs ID50 nAbs (A) and RBD IgG arbitrary units (AU) (B). Subjects naïve for COVID-19 were stratified as above or below the median of HKU1 S2 IgGs at day 10 post-vaccination. Filled circles and bars represent the median \pm inter quartile range at each time-point, and

RBD [14]. Therefore, caution should be exercised in using $\mathrm{RBD}$ antibodies as proxy for $\mathrm{VoC}$ neutralization or as a surrogate marker to predict vaccine effectiveness. Essentially all current large-scale screening immunoassays that measure RBD antibodies are based on the Wuhan-Hu-1 sequence. Our study suggests that simply switching the RBD antigen sequence to that of VoCs would not lead to an efficient detection of differences in neutralizing activity among screened sera.

Furthermore, our data shows that BNT162b2 vaccination frequently induces an early boost of antibodies to the $\mathrm{S} 2$ subunit of OC43 and HKU1 seasonal betacoronaviruses. This boost was rapid and large in $>50 \%$ of vaccinees naïve for SARS-CoV-2 infection. Interestingly, the rapid increase of $\mathrm{IgG}$ to HKU1 at 10 days post-mRNA vaccination was associated with a trend to faster decrease and subsequent loss of SARS-CoV-2-specific antibodies. While a confirmation in a larger cohort is warranted in light of the limited number of subjects analyzed in this study, in naïve vaccinees, a strong reactivation of pre-existing seasonal coronaviruses antibody responses was associated with a statistically significant subsequent reduction of nAbs against the empty circles show each individual measurement. The horizontal dashed lines stand for the threshold for positivity. The asterisks indicate statistically significant differences in mean ID50 nAbs or RBD IgG AU at the corresponding time-points between subjects with or without an early boost of seasonal coronaviruses responses after vaccination (two-way repeated measures ANOVA, $* p<0.05, * * p<0.01$ )

alpha and beta VoCs. In the case of the beta VoC, which is currently thought as the most immune evasive [15], this was also associated with a rapid loss of neutralization.

We previously described an increase of antibodies against HKU1 and OC43 Spike antigens in a cohort of symptomatic COVID-19 patients shortly after disease onset [9]. In those patients, HKU1 and OC43 antibody levels were significantly correlated with those of SARS-CoV-2-specific antibodies during the first 3-4 weeks from symptoms onset. The existence of cross-reactive antibodies between SARS-CoV-2 and seasonal betacoronaviruses has been demonstrated also by other studies $[16,17]$ and further supported by the isolation of cross-reactive monoclonal antibodies from germinal centers of vaccinated individuals [14]. The coding sequence of these cross-reactive monoclonal had more extensive somatic mutations compared to non-cross-reactive ones, signaling a likely derivation from memory responses pre-existing vaccination. Moreover, these findings are consistent with the existence of betacoronaviruses cross-reactive $\mathrm{T}$ cells in subjects that were either naïve for SARS-CoV-2 infection or had COVID-19 [2, $18,19]$. 
It is relatively straightforward to speculate that the observed early rapid expansion of HKU1 and OC43 IgG antibodies post-vaccination could be attributed to the presence of both elevated pre-existing memory B cells to seasonal coronaviruses and cross-reactive helper $\mathrm{T}$ cells recognizing peptide epitopes in the Spike S2 subunit, due to its relatively high homology between coronaviruses. However, it is unclear how the expansion of memory responses to other betacoronaviruses Spike S2 might subsequently impact on SARS-CoV-2 nAbs development. The $\mathrm{S} 2$ subunit is rarely if ever directly targeted by SARS-CoV-2 nAbs [20]. It is therefore unlikely that an early deviation of antibody responses toward the $\mathrm{S} 2$ of seasonal betacoronaviruses rather than that of SARS-CoV-2 might lead to a direct decrease of nAbs. Furthermore, our data suggest also a partially generalized reduction of SARS-CoV2 -specific antibodies including binding $\operatorname{IgG}$ against the spike RBD in naïve subjects with an early reactivation of seasonal betacoronaviruses' B cell memory. Possibly, this observation might support the concept of a preferential and synchronous expansion of a subset of pre-existing and cross-reactive $T$ cells recognizing $\mathrm{S} 2$ rather than $\mathrm{S} 1$ epitopes.

The persistence of nAbs is key for protection from SARS$\mathrm{CoV}-2$ infection. However, modeling studies based on immunological data from mild and moderate COVID-19 patients suggest that nAbs elicited from less severe natural infection is unlikely to provide long-term protection from infection [21]. Our own data suggests that the re-expansion of pre-existing humoral memory to seasonal betacoronaviruses might dampen the SARS-CoV-2 neutralizing response induced by vaccination. Potentially, this would imply that in a relevant fraction of vaccinees naïve for COVID-19, the duration of protection from subsequent re-infection would be shortened. Additionally, it might be speculated that this mechanism might be at play also in individuals who developed SARSCoV-2 antibodies upon infection, a fact that might contribute to the waning of SARS-CoV-2 nAbs that we observed in our study in some individuals who had an asymptomatic COVID19 before vaccination.

While the effectiveness of the immunization with current mRNA vaccines is undisputable in reducing both infection and disease severity [22], public health policies must adapt to rapidly emerging VoCs. The choice of diagnostic tests, vaccine formulations, and vaccine deployment will benefit from investigating further the mechanisms influencing the response to vaccination, particularly in view of the ongoing development of pan-coronavirus vaccines [23].

Supplementary Information The online version contains supplementary material available at https://doi.org/10.1007/s10875-021-01190-5.

Acknowledgements We thank Foundation Dormeur, Vaduz for the donation of laboratory instruments relevant to this project to the Viral Evolution and Transmission Unit at OSR and to the National Center for Global Health at the Istituto Superiore di Sanità. A special acknowledgement goes to the COVID-BioB team and health care workers at OSR that made this work possible. The sponsors had no role in study design; in the collection, analysis, and interpretation of data; in the writing of the report; and in the decision to submit the paper for publication.

Author Contribution S. Dispinseri and I. Marzinotto share the first authorship in this paper, and their order was chosen alphabetically. Correspondence to Vito Lampasona (lampasona.vito@hsr.it) and Gabriella Scarlatti (scarlatti.gabriella@ hsr.it).GS, VL, ACara, and PC conceived and designed the study. GS, SD, and VL wrote the first draft with contributions from ACara, DN, and LP. VL, IM, and GS did the statistical analysis. SD, MT, CB, EB, MP, PMK, ACan, MB, and AG did the laboratory tests. SD, IM, VL, and GS did the data organization, data storage, and quality control. SD, RC, RV, and CB oversaw sample handling and storage. GS, FC, and PC oversaw institutional review board submissions, approval, and clinical study oversight. GS, LP, VL, FC, ACara, and DN provided funding. VL, GS, DN, ACara, IM, and SD prepared the manuscript for submission. VL and GS jointly supervised this work. All authors reviewed the manuscript for intellectual content and assisted in data interpretation. All authors confirm that they had full access to all the data in the study and accept responsibility to submit for publication.

Funding The work performed at IRCCS Ospedale San Raffaele (OSR) was funded by Program Project COVID-19 OSR-UniSR and Ministero della Salute (COVID-2020-12371617). The work by Viral Evolution and Transmission Unit, OSR, Istituto Superiore di Sanità (ISS), and Imperial College was funded by EAVI2020, the European Union's Horizon 2020 research and innovation programme under grant agreement No. 681137. ISS received support in part by NATO multi-year Project No. G5817 "New and Validated Tools for the Diagnosis and follow-up of SARS-CoV-2 Infected Individuals" and by ISS internal funds.

Availability of Data and Material The data generated in the study might be made available upon reasonable request.

Code Availability Not applicable.

\section{Declarations}

Ethics Approval All procedures performed in studies involving human participants were in accordance with the ethical standards of the institutional and/or national research committee and with the 1964 Helsinki declaration and its later amendments or comparable ethical standards. The study subjects belonged to the IRCCS Ospedale San Raffaele COVID-19 cohort study COVID-BioB (registered as ClinicalTrialsgovNCT04318366) and approved by the IRCCS Ospedale San Raffaele Ethics Review Board (protocol 68/INT/2020) and to the COVID-BioB related immunological sub-study "Role of the immune response in the infection with SARS-CoV-2 and in the pathogenesis of COVID-19" (protocol number 34/INT/2020).

Consent to Participate Written informed consent was obtained from all individual participants included in the study.

Consent for Publication Not applicable.

Conflict of Interest LP and VL have a patent pending that refers to polypeptides, nucleic acids, vectors, and host cells and their use in the diagnosis and/or treatment of COVID-19 infections. All other authors have declared that no conflict of interest exists. 
Open Access This article is licensed under a Creative Commons Attribution 4.0 International License, which permits use, sharing, adaptation, distribution and reproduction in any medium or format, as long as you give appropriate credit to the original author(s) and the source, provide a link to the Creative Commons licence, and indicate if changes were made. The images or other third party material in this article are included in the article's Creative Commons licence, unless indicated otherwise in a credit line to the material. If material is not included in the article's Creative Commons licence and your intended use is not permitted by statutory regulation or exceeds the permitted use, you will need to obtain permission directly from the copyright holder. To view a copy of this licence, visit http://creativecommons.org/licenses/by/4.0/.

\section{References}

1. Abecassis A. Five priorities for universal COVID-19 vaccination. The Lancet [Internet]. 2021 [cited 2021 Aug 6];398:285-6. Available from: https://linkinghub.elsevier.com/retrieve/pii/S0140673621013714

2. Grifoni A, Weiskopf D, Ramirez SI, Mateus J, Dan JM, Moderbacher CR, et al. Targets of $\mathrm{T}$ cell responses to SARS-CoV-2 coronavirus in humans with COVID-19 disease and unexposed individuals. Cell [Internet]. 2020 [cited 2020 Jul 15];181:1489_ 1501.e15. Available from: https://linkinghub.elsevier.com/retri eve/pii/S0092867420306103

3. Harvey WT, Carabelli AM, Jackson B, Gupta RK, Thomson EC, Harrison EM, et al. SARS-CoV-2 variants, spike mutations and immune escape. Nat Rev Microbiol [Internet]. 2021 [cited 2021 Jul 7];19:409-24. Available from: http://www.nature.com/artic les/s41579-021-00573-0

4. Cohen J. Can immune responses alone reveal which COVID-19 vaccines work best? [Internet]. Science I AAAS. 2021 [cited 2021 Aug 6]. Available from: https://www.sciencemag.org/news/2021/ 07/can-immune-responses-alone-reveal-which-covid-19-vacci nes-work-best

5. High demand for the third COVID vaccine dose: more than a quarter of a million people have already been vaccinated [Internet]. GOV.IL. [cited 2021 Aug 6]. Available from: https://www. gov.il/en/departments/news/05082021-01

6. Dispinseri S, Secchi M, Pirillo MF, Tolazzi M, Borghi M, Brigatti C, et al. Neutralizing antibody responses to SARS-CoV-2 in symptomatic COVID-19 is persistent and critical for survival. Nat Commun [Internet]. 2021 [cited 2021 May 17];12:2670. Available from: http://www.nature.com/articles/s41467-021-22958-8

7. Fenyö EM, Heath A, Dispinseri S, Holmes H, Lusso P, Zolla-Pazner $\mathrm{S}$, et al. International network for comparison of HIV neutralization assays: the NeutNet report. Unutmaz D, editor. PLoS ONE [Internet]. 2009 [cited 2021 Aug 6];4:e4505. Available from: https:// dx.plos.org/https://doi.org/10.1371/journal.pone.0004505

8. Burbelo PD, Goldman R, Mattson TL. A simplified immunoprecipitation method for quantitatively measuring antibody responses in clinical sera samples by using mammalian-produced Renilla luciferase-antigen fusion proteins. BMC Biotechnol. 2005;5:22.

9. Secchi M, Bazzigaluppi E, Brigatti C, Marzinotto I, Tresoldi C, Rovere-Querini P, et al. COVID-19 survival associates with the immunoglobulin response to the SARS-CoV-2 spike receptor binding domain. Journal of Clinical Investigation [Internet]. 2020 [cited 2021 Jul 9];130:6366-78. Available from: https://www.jci.org/artic les/view/142804

10. Lind A, Marzinotto I, Brigatti C, Ramelius A, Piemonti L, Lampasona V. A/H1N1 hemagglutinin antibodies show comparable affinity in vaccine-related Narcolepsy type 1 and control and are unlikely to contribute to pathogenesis. Scientific Reports [Internet]. 2021;11. Available from: https://www.scopus.com/inward/ record.uri?eid=2-s2.0-85101199440\&doi $=10.1038 \% 2$ fs41598021-83543-z\&partnerID=40\&md5=f16c1b011fb1d2e4e6bc208ed 5986663

11. Dominguez SR, Shrivastava S, Berglund A, Qian Z, Góes LGB, Halpin RA, et al. Isolation, propagation, genome analysis and epidemiology of HKU1 betacoronaviruses. J Gen Virol [Internet]. 2014 [cited 2021 Oct 29];95:836-48. Available from: https:// www.ncbi.nlm.nih.gov/pmc/articles/PMC3973476/

12. R Core Team. R: a language and environment for statistical computing [Internet]. Vienna, Austria: R Foundation for Statistical Computing; 2020. Available from: https://www.R-project.org/

13. Pouwels KB, Pritchard E, Matthews PC, Stoesser N, Eyre DW, Vihta K-D, et al. Impact of Delta on viral burden and vaccine effectiveness against new SARS-CoV-2 infections in the UK [Internet]. Epidemiology; 2021 Aug. Available from: http:// medrxiv.org/lookup/doi/https://doi.org/10.1101/2021.08.18.21262 237

14. Amanat F, Thapa M, Lei T, Ahmed SMS, Adelsberg DC, Carreño JM, et al. SARS-CoV-2 mRNA vaccination induces functionally diverse antibodies to NTD, RBD, and S2. Cell [Internet]. 2021 [cited 2021 Jul 9];S0092867421007066. Available from: https:// linkinghub.elsevier.com/retrieve/pii/S0092867421007066

15. Lazarevic I, Pravica V, Miljanovic D, Cupic M. Immune evasion of SARS-CoV-2 emerging variants: what have we learnt so far? Viruses [Internet]. 2021 [cited 2021 Nov 19];13:1192. Available from: https://www.mdpi.com/1999-4915/13/7/1192

16. Kaplonek P, Wang C, Bartsch Y, Fischinger S, Gorman MJ, Bowman $\mathrm{K}$, et al. Early cross-coronavirus reactive signatures of protective humoral immunity against COVID-19 [Internet]. Immunology; 2021 May. Available from: http://biorxiv.org/lookup/ doi/https://doi.org/10.1101/2021.05.11.443609

17. Hicks J, Klumpp-Thomas C, Kalish H, Shunmugavel A, Mehalko J, Denson J-P, et al. Serologic cross-reactivity of SARS-CoV-2 with endemic and seasonal betacoronaviruses. J Clin Immunol [Internet]. 2021 [cited 2021 Aug 5];41:906-13. Available from: https://link.springer.com/https://doi.org/10.1007/ s10875-021-00997-6

18. Woldemeskel BA, Kwaa AK, Garliss CC, Laeyendecker O, Ray SC, Blankson JN. Healthy donor T cell responses to common cold coronaviruses and SARS-CoV-2. Journal of Clinical Investigation [Internet]. 2020 [cited 2021 Aug 5];130:6631-8. Available from: https://www.jci.org/articles/view/143120

19. Braun J, Loyal L, Frentsch M, Wendisch D, Georg P, Kurth F, et al. SARS-CoV-2-reactive T cells in healthy donors and patients with COVID-19. Nature [Internet]. Nature Publishing Group; 2020 [cited 2020 Aug 4];1-8. Available from: http://www.nature. com/articles/s41586-020-2598-9

20. Graham C, Seow J, Huettner I, Khan H, Kouphou N, Acors S, et al. Neutralization potency of monoclonal antibodies recognizing dominant and subdominant epitopes on SARS-CoV-2 Spike is impacted by the B.1.1.7 variant. Immunity [Internet]. 2021 [cited 2021 Aug 5];54:1276-1289.e6. Available from: https:// linkinghub.elsevier.com/retrieve/pii/S1074761321001357

21. Wheatley AK, Juno JA, Wang JJ, Selva KJ, Reynaldi A, Tan H-X, et al. Evolution of immune responses to SARS-CoV-2 in mildmoderate COVID-19. Nat Commun [Internet]. 2021 [cited 2021 Aug 5];12:1162. Available from: http://www.nature.com/articles/ s41467-021-21444-5

22. Haas EJ, Angulo FJ, McLaughlin JM, Anis E, Singer SR, Khan $\mathrm{F}$, et al. Impact and effectiveness of mRNA BNT162b2 vaccine against SARS-CoV-2 infections and COVID-19 cases, hospitalisations, and deaths following a nationwide vaccination campaign in Israel: an observational study using national surveillance data. The Lancet [Internet]. 2021 [cited 2021 May 17];397:1819-29. Available from: https://linkinghub.elsevier.com/retrieve/pii/S0140 673621009478 
23. Shiakolas AR, Kramer KJ, Wrapp D, Richardson SI, Schäfer A, Wall S, et al. Cross-reactive coronavirus antibodies with diverse epitope specificities and Fc effector functions. Cell Reports Medicine [Internet]. 2021 [cited 2021 Jul 7];2:100313. Available from: https://linkinghub.elsevier.com/retrieve/pii/S2666379121001567
Publisher's Note Springer Nature remains neutral with regard to jurisdictional claims in published maps and institutional affiliations. 